\title{
Further data on degree of learning and transfer of training'
}

\author{
L. R. GOULET, RICHARD A. MELTZER, AND KATHLEEN K. O'SHAUNESSY
}

WEST VIRGINIA UNIVERSITY

Transfer of training as a function of first-list learning was studied by varying both the number of List 1 practice trials (9 trials, 18 trials, trials to one perfect repetition plus 9 overlearning trials) and the degree of learning of individual pairs within the list. The degree of learning of individual pairs on the first list was manipulated by incorporating the \% OSM technique of partial reinforcement (100, 67, and $33 \%$ OSM). The results indicated that negative transfer in the $A-B, A-C$ paradigm (as compared to the $A-B, C-D$ control paradigm) was directly related to the number of practice trials but was not related to \% OSM level. These results substantially replicated an earlier study by Goulet who used the same paradigms and varied the number of practice trials and \% ORM partial reinforcement.

There have been a number of recent studies concerned with the effects of the degree of List 1 learning on transfer of training under the $A-B, A-C$ paradigm (Goulet, 1967; Jung, 1962; Postman, 1962). The results of this work generally suggest that the negative transfer observed with this paradigm increases directly with the number of List 1 practice trials as long as no overlearning trials are given. However, when practice is continued beyond mastery the negative transfer decreases (Jung, 1962; Postman, 1962).

Goulet (1967) has noted a potential source of confounding in previous work. This source of confounding is related to the possible interaction between individual pair overlearning and the number of List 1 practice trials. That is, when practice is discontinued prior to List 1 mastery, the easiest pairs are learned or even overlearned, with the more difficult pairs not being anticipated correctly. Goulet (1967) attempted to separate the effects of the two sources of transfer, the effects of List 1 practice qua practice and pair overlearning, as they each contribute to the List 1 learning-transfer function. Differential learning of individual pairs in the list was manipulated by incorporating the \% ORM (percentage of occurrence of response members) partial reinforcement methodology. This technique involves deleting the response terms on a fixed percentage of the learning trials, a procedure which effectively retards both response learning and associative learning in direct proportion to the percentage of trials that the responses are deleted. Varying \% ORM with a mixed list assures differential degrees of learning for individual pairs while still permitting the number of practice trials to be held constant. Contrary to expectation, Goulet (1967) found that the number of practice trials, rather than the degree of learning (or overlearning) of the individual pairs, was the major factor contributing to the List 1 learningtransfer function. In fact, the negative transfer observed with this paradigm was independent of List $1 \%$ ORM level.

The present experiment extended that of Goulet (1967) by varying \% OSM rather than \% ORM. The \% OSM methodology involves deleting the stimulus terms on a fixed percentage of the trials, a procedure which retards only associative learning (Goulet, 1966; Schulz, 1965) as long as Ss are forced to attend to the responses on trials where the stimuli are deleted (Goulet, 1966; Goulet \& Solso, 1966). Degree of List 1 practice (learning?) was manipulated in the present experiment by varying both the number of practice trials (nine trials, 18 trials or one perfect repetition of the list, one perfect repetition plus nine trials) and List $1 \%$ OSM level $(100 \%, 67 \%, 33 \%)$. As will be noted, the present experiment included a treatment which involved List 1 overlearning in contrast to that of Goulet (1967), where List 1 practice varied from minimal degrees of learning to list mastery (one perfect repetition).

\section{Method}

Design. The basic design was a 3 by 2 by 3 mixed factorial with List $1 \%$ OSM as a mixed-list factor and paradigm $(A-B, A-C$, experimental; $A-B, C-D$, control) and List 1 practice $(9 \mathrm{~T}, 18 \mathrm{~T}, 1 \mathrm{P}+9 \mathrm{~T})$ as between-S factors. Partial reinforcement was manipulated such that three pairs each in List 1 were randomly assigned to the 33,67 , and $100 \% \mathrm{OSM}$ levels. The stimuli under $33 \%$ OSM were paired with responses once per block of three trials, while those in the 67 and 100\% OSM levels were paired two and three times per block of three trials, respectively. To control for pair difficulty three sets of lists were constructed for each paradigm. The alternate lists permitted each List 1 pair to be assigned to the 33 , 67 , or $100 \%$ OSM levels in different lists. All lists consisted of nine paired adjectives (low similarity) taken from Haagen (1949). List 2 was identical for all groups and did not involve partial reinforcement.

Procedure. The Ss were 108 undergraduates who were randomly assigned to treatments with the restriction that equal numbers be assigned to each treatment before another replication block was begun. List 1 was presented using the training-test (blocking) method with a 2-sec presentation of each pair during training and a 2-sec anticipation interval during the 
Table 1. Mean Correct Anticipations on Trials 1-5

\begin{tabular}{|c|c|c|c|c|c|c|}
\hline & \multirow[b]{3}{*}{$33 \%$ ORM } & \multicolumn{4}{|c|}{ Paradigm } \\
\hline & & & \multicolumn{3}{|c|}{ D } & \multirow{2}{*}{$\begin{array}{r}\text { A-B,C-D } \\
\text { Total }\end{array}$} \\
\hline & & & $67 \%$ ORM & $100 \% 0 \mathrm{RM}$ & Total & \\
\hline \multirow{3}{*}{$\begin{array}{l}\text { List } 1 \\
\text { Practice }\end{array}$} & $9 T$ & 5.44 & 5.22 & 4.72 & 15.39 & 19.78 \\
\hline & $18 \mathrm{~T}$ & 4.83 & 5.89 & 4.72 & 15.44 & 20. \\
\hline & $1 P+9$ & 5.11 & 6.00 & 5.67 & 16.78 & 23.22 \\
\hline
\end{tabular}

test half of the trial. List 2 involved the anticipation method and a 2:2-sec rate. All lists were presented on a Stowe memory drum in six random orders with a $4-\mathrm{sec}$ intertrial interval. List 2 was practiced for 10 trials or to a criterion of one perfect trial, whichever occurred last. To assure attention to the responses when the stimuli were deleted (Goulet, 1966) the Ss were asked to pronounce all words as soon as they appeared in the window of the drum.

\section{Results and Discussion}

List $I$ and List 2 Learning. List 1 learning varied directly with \% OSM level. Inspection of the data revealed essentially identical rates of learning for all groups on Trials 1-9 (where data were available for all Ss). The list was learned to a criterion of one perfect trial in 16.05 and 17.94 trials, for the $1 \mathrm{P}+9 \mathrm{E}$ and $\mathrm{C}$ treatments, respectively.

Table 1 provides summary data for List 2 learning at each level of List 1 practice and $\%$ OSM level. For the A-B, C-D paradigm, the means represent correct anticipations for the entire nine-pair list, independently of List $1 \%$ OSM level since the List 2 pairs could not legitimately be identified with a particular List $1 \%$ OSM level. The parallel data are presented for the A-B, A-C paradigm in addition to presenting correct anticipations on Trials $1-5$ for the pairs identified with the List $1,33,67$, and $100 \%$ OSM levels.

The first analysis involved a comparison of correct anticipations on Trials $1-5$ between the A-B, A-C and $A-B, C-D$ paradigms (pooled over \% OSM). The E/C and practice main effects were statistically significant $(F=16.08, d f=1 / 102, p<.001$ and $F=3.41, d f=2 / 102, p$ $<.05)$. Learning was better under the $A-B, C-D$ paradigm than under the A-B, A-C paradigm; i.e., negative transfer occurred at each level of List 1 practice. Rate of learning also increased directly as a function of the number of List 1 practice trials although this tendency was more apparent under the A-B, C-D paradigm. The practice by $\mathrm{E} / \mathrm{C}$ interaction, however, failed to reach statistical significance ( $F=1.74$, df $=2 / 102, p>.10)$. The practice effect presumably reflects greater learning-to-learn transfer for the treatments which had the greater number of List 1 practice trials. The reduced effect under the A-B, A-C paradigm may be accounted for by a counterbalancing of nonspecific positive (learning-to-learn) and specific negative (associative interference) sources of transfer.

The effects of $\%$ OSM on transfer under the A-B, A-C paradigm were also determined. Difference scores were obtained by subtracting the mean correct anticipations/ 3 on Trials 1-5 under the A-B, C-D paradigm from each S's score for each level of \% OSM under the $A-B, A-C$ paradigm. This was done for each level of List 1 practice. In this analysis only the main effect of practice attained statistical significance $(F=3.64$, $\mathrm{df}=2 / 51, \mathrm{p}<.05)$. These data indicated that negative transfer increased directly with the number of List 1 practice trials.

The final analysis was concerned with the effects of List $1 \%$ OSM on learning under the A-B, A-C paradigm independently of the rate of learning for the A-B, C-D paradigm. Neither main effect (List 1 practice or List $1 \%$ OSM) nor the interaction approached statistical significance ( $F<1, \mathrm{df}=2 / 51, \mathrm{~F}$ $=1.61, \mathrm{df}=2 / 102, \mathrm{~F}<1, \mathrm{df}=4 / 102$, respectively). List 2 learning under the $A-B, A-C$ paradigm was independent of both \% OSM and level of List 1 practice. These results generally confirm those obtained by Postman (1962) and Goulet (1967), who found that rate of learning under the $A-B, A-C$ paradigm was independent of the number of List 1 practice trials. In addition, the data of the present study and those of Goulet (1967) suggest that the number of practice trials, rather than degree of learning of the individual pairs, is the primary factor responsible for the direct relation between negative transfer and degree of first-list practice found in previous research.

\section{References}

GOULET, L. R. Associative and free recall of paired-associates under conditions of response articulation and \% OSM partial reinforcement. Psychon. Sci, 1966, 5, 227-228.

GOULET, L. R. Degree of learning and transfer of training. Psychon. Sci, 1967, 8, 245-246.

GOULET, L. R., \& SOLSO, R. L. Percentage occurrence of stimulus members and meaningfulness as related to forward and backward recall of paired-associates. J. exp. Psychol, 1966, 71, 494-498.

HAAGEN, C. H. Synonymity, vividness, familiarity and association values for 400 pairs of common adjectives. $J$. Psychol, 1949, 30, 185-200.

JUNG, J. Transfer of training as a function of degree of first-list learning. J. verbal Learn verbal Behav., 1962, 1, 197-199.

POSTMAN, L. Transfer of training as a function of experimental paradigm and degree of first-list learning. J. verbal Learm verbal Behav., 1962, 1, 109-118.

SCHULZ, R. W. Learning of paired-associates as a function of pronounciability and percentage occurrence of stimulus members. $J$. verbal Learn. verbal Behav., 1965, 4, 494497.

\section{Note}

1. The present research was supported by NIMH Grant $13130-01$ to the first author. 